Bangladesh J. Bot. 48(4): 1083-1089, 2019 (December)

\title{
COMPLETE CHLOROPLAST GENOME SEQUENCE OF A VEGETATIVE BERMUDAGRASS CULTIVAR 'TIFEAGLE' (CYNODON DACTYLON $\times$ CYNODON TRANSVAALENSIS)
}

\author{
Yancai Shi, Miao Chen ${ }^{1,2}$, Shaofeng Jiang ${ }^{3}$ and Shilian Huang ${ }^{4}$ \\ Guangxi Institute of Botany, Chinese Academy of Sciences, Guilin, China
}

Keywords: Cynodondactylon $\times$ C. transvaalensis, Chloroplast genome, Repeat sequence, Germplast generation, Conservation genetic, Collinear analysis

\begin{abstract}
Hybrid (Cynodonn dactylon $\times$ C. transvaalensis) is a widely distributed turfgrass and shows a great value of environment, horticulture and economic. Though, the chloroplast genome of $C$. dactylon has been reported, it might be helpful finding reasons that triploid bermudagrass shows a better drought and trampling tolerance than common bermudagrass through comparing chloroplast genome analysis. The present results showed the complete chloroplast genome of the $C$. dactylon $\times C$. transvaalensis is $134655 \mathrm{bp}$ in length. The tetramerous genome contained a large single copy (LSC) region (79,998 bp), a small single copy (SSC) region $(12,517 \mathrm{bp})$, and a pair of inverted repeat (IR) regions $(42,140 \mathrm{bp})$. In the chloroplast genome, 116 genes were predicted, including 83 protein-coding, 29 tRNA and 4 rRNA genes. Furthermore, a total of 80 repeat sequences were identified. Only $0.23 \%$ intergenicnon-collinear sequences were found between the chloroplast genome of Cynodon dactylon $\times$ C. transvaalensis and Cynodon dactylon.
\end{abstract}

\section{Introduction}

Cynodon dactylon $\times$ Cynodon transvaalensis is widely used in sports fields, lawns, parks, golf courses (Harlan et al. 1970). The triploid hybrid bermudagrass showed higher turf quality with limited irrigation than common bermudagrass (Hanna 1998). Hu et al. $(2009,2010)$ reported that the better ability of drought tolerance due to the hybrid bermudagrass had more active ribulose-1,5-bisphosphate carboxylase/oxygenase (RuBisCO) enzyme, RuBisCO activase and more stable proteins for carbon assimilation which made a greater capability of photosynthesis. By comparative proteomic analyzing the difference between triploid and common bermudagrass under water-deficit stress, Zhao et al. (2011) found a inhibition of expression of Chl a-b binding proteins, oxygen-evolving enhancer protein, ATP synthase and RuBisCO large subunit in common bermudagrass under drought stress. These results demonstrate there exist a differential gene expression and enzymatic activity in chloroplast under drought stress between triploid and common bermudagrass. It is speculated that chloroplast sequence variation happened in the process of triploid bermudagrass formation. If so, the genomic resources of the sequence of $C$. dactylon (Genbank accession number KY024482.1) (Huang et al. 2017) and hybrid (C. dactylon $\times$ C. transvaalensis) will be of great value for new bermudagrass germplasm generation and genetic conservation.

\footnotetext{
*Author for correspondence: <shiil_huang@163.com>. ${ }^{1}$ Faculty of Agricultural Science, Guangdong Ocean University, Zhanjiang, P. R. China. ${ }^{2}$ State Key Laboratory for Conservation and Utilization of Subtropical Agro-bioresources; Guangdong Engineering Research Center for Grassland Science; College of Life Sciences, South China Agricultural University, Guangzhou, Guangdong, China. ${ }^{3}$ Guilin Medical University,Guilin, Guangxi, China. ${ }^{4}$ College of Life Sciences, South China Agricultural University, Guangzhou, China.
} 


\section{Materials and Methods}

Fresh plant was collected from Laboratory for Turfgrass Science, College of Life Science, South China Agricultural University. The genomic DNA was extracted according to the method of CTAB (Porebski et al. 1997). The quality and concentration of the DNA products were assessed using agarose gel electrophoresis and a NANODROP 1000 spectrophotometer (Thermo Scientific).

Fifteen pair of primers (Table 1) was designed based on Cynodon dactylon, according to the method mentioned by Zhang et al. (2016). Amplification of target gene regions was carried out using the PCR in Eppendorf Mastercycler nexus (Hamburg, Germany).

Table 1. Sequences of 15 pairs of primers used for Cynodon dactylon $\times$ C. transvaalensis chloroplast genome amplification.

\begin{tabular}{ll}
\hline Primername & Primer sequence \\
\hline CPUP_1F & GCACTTAAAAGCCGAGTACTCTACCA \\
CPUP_1R & CAAAGGTTTAGAAGACCTCTGTCCTATCCA \\
CPUP_2F & CCATTGTCTAATGGATAGGACAGAGGTC \\
CPUP_2R & AGGACAAATGATTGATTTACCTATTCAAAG \\
CPUP_3F & TGCTTTGAATAGGTAAATCAATCATTTGT \\
CPUP_3R & CTGTCAAGGCGGAAGCTGCGGG \\
CPUP_4F & GAACCCGCAGCTTCCGCCTTGAC \\
CPUP_4R & TTAAAAGTTGCTCCTGCTACTCAGCC \\
CPUP_5F & CAAGGCTCTAGGCTGAGTAGCAGGAG \\
CPUP_5R & ACCAGATTTGAACTGGTGACACGAGGA \\
CPUP_6F & ACTGAAAATCCTCGTGTCACCAGTTCA \\
CPUP_6R & ATGCATACCATGATTTTTCTGTCTATCA \\
CPUP_7F & GAGCAATGCATGCAGTTATTGATAGA \\
CPUP_7R & AGGTTCAAATCCTACAGAGCGTGAT \\
CPUP_8F & ATCACGCTCTGTAGGATTTGAACC \\
CPUP_8R & TTAATAATTCAAGTCACACACTCCCA \\
CPUP_9F & TGGGAGTGTGTGACTTGAATTATTAATT \\
CPUP_9R & ATCCATGGCTGAATGGTTAAAGCGCC \\
CPUP_10F & TTTACCAATTATGAGTTGGGCGCTTT \\
CPUP_10R & AGGAAGAGCACTTGCCATTCGTTGGT \\
CPUP_11F & CCATATTTCGACCCGGACGCTTTTGG \\
CPUP_11R & GATGCCTCAGCTGCATACATCACTGC \\
CPUP_12F & ATGCAGCTGAGGCATCCTAACGAACG \\
CPUP_12R & TGCTTCCTAAGAGCAGCGTGTCTACC \\
CPUP_13F & GGTAGACACGCTGCTCTTAGGAAG \\
CPUP_13R & CACCAATAAGATACGGAGACTTGCTTCACA \\
CPUP_14F & TGTGTAATTCCAAATGTGAAGCAAGTCT \\
CPUP_14R & GGTTCGAATCCCTCCTCGCCCA \\
CPUP_15F & ATCCATGGCTGAATGGTTAAAGCGCC \\
CPUP_15R & CTCAATGGTAGAGTACTCGGCTT \\
\hline &
\end{tabular}


PCR product was extracted with the SDS method. The harvested DNA was detected by the agarose gel electrophoresis and quantified by Qubit. Whole-genome sequencing was performed on the IlluminaHiSeq 2500-PE125 platform with MPS (massively parallel sequencing) Illumina technology. A-tailed, ligated to paired-end adaptors and PCR amplified with a $500 \mathrm{bp}$ insert and a mate-pair library with an insert size of $5 \mathrm{~kb}$ were used for the library construction at the Beijing Novogene Bioinformatics Technology Co., Ltd.

Illumina PCR adapter reads and low-quality reads from the paired-end and matepair library were filtered by the step of quality control using our own compling pipeline. All good quality paired reads were assembled using the SOAPdenovo (Li et al. 2008, 2010) (http://soap. genomics.org.cn/soapdenovo.html) into several scaffolds. Then the filter reads were handled by the next step of the gap-closing.

Preliminary gene prediction was performed with the online program DOGMA (Wyman et al. 2004). All tRNA genes were predicted by tRNA scanSE search server (Schattner et al. 2005). Repeats were detected by Tandem Repeats Finder (Department of Biomathematical Sciences, New York, NY, USA) (https://tandem.bu.edu/trf/trf.html) and REPuter (Benson 1999 and Kurtz et al. 2001). Annotated genome was submitted to online server Organellar GenomeDRAW for visualization (Lohse et al. 2013).

The blast results between triploid and common bermudagrass chloroplast genome were inputted into MCscan56 with default parameters to compute multiple synteny. The final gene collinearity results were generated from the MCscan output file by swapping the gene order number of each gene with their names using a Perl script.

\section{Results and Discussion}

The complete chloroplast genome of the Cynodon dactylon $\times$ C. transvaalensis is $134655 \mathrm{bp}$ in length. The whole genome sequence data reported in this paper have been deposited in the Genome Warehouse in BIG data center (2017), Beijing Institute of Genomics (BIG), Chinese Academy of Sciences, under accession number GWHAAAN00000000 that is publicly accessible at http://bigd.big.ac.cn/gwh. The genome contains a pair of inverted repeat (IR) regions (42, 140 bp), which separated by two single copy regions (LSC 79, 998 bp and SSC 12, $517 \mathrm{bp).} \mathrm{The} \mathrm{GC}$ content of the sequence is $38.38 \%$, with the corresponding values of $36.3,32.58,44.03 \%$ for the LSC, SSC and IR regions, respectively. In the chloroplast genome, there are 116 genes predicted, including 83 protein-coding, 29 tRNA and 4 rRNA genes (Table 2). A total of 80 repeats were identified in the chloroplast genome of Cynodon dactylon $\times$ C. transvaalensis, containing 21 forward repeats, 26 palindromic repeats, 3 reverse repeats and 30 tandem repeats. The length of repeats mainly lies between 21 and $30 \mathrm{bp}(53.75 \%)$. The tandem repeats have a wide length range and the length of forward and palindromic repeats mostly lies between 21 and $50 \mathrm{bp}$.

From the results of the collinear analysis, the two genomes are almost identical and only $0.23 \%$ of them are non-collinear (Fig. 2), which are intergenic. Also, sequences of genes associated with photosynthesis were compared and only three different bases were found in $n d h \mathrm{~A}$ (TCC/TCT), psaA (GTT/GTA) and psaJ (CCC/CCG). However, they encode the same amino acids, TCC and TCT encode serine, GTT and GTA encode valine, CCC and CCG encode proline.

It is well known that chloroplast gene is matrilineal inheritance. Theoretically, Cynodon dactylon $\times$ C. transvaalensis and Cynodon dactylon have the same chloroplast genome. The present hypothesis is based on the chloroplast genome variation in the process of triploid bermudagrass formation. Comparative analysis result demonstrated that there was almost no difference between the chloroplast genome between Cynodon dactylon $\times$ C. transvaalensis and Cynodon dactylon. The higher expression of photosynthesis-related genes, more active RuBisCO 
enzyme, and stable proteins may occur mainly due to the different nuclear genome.

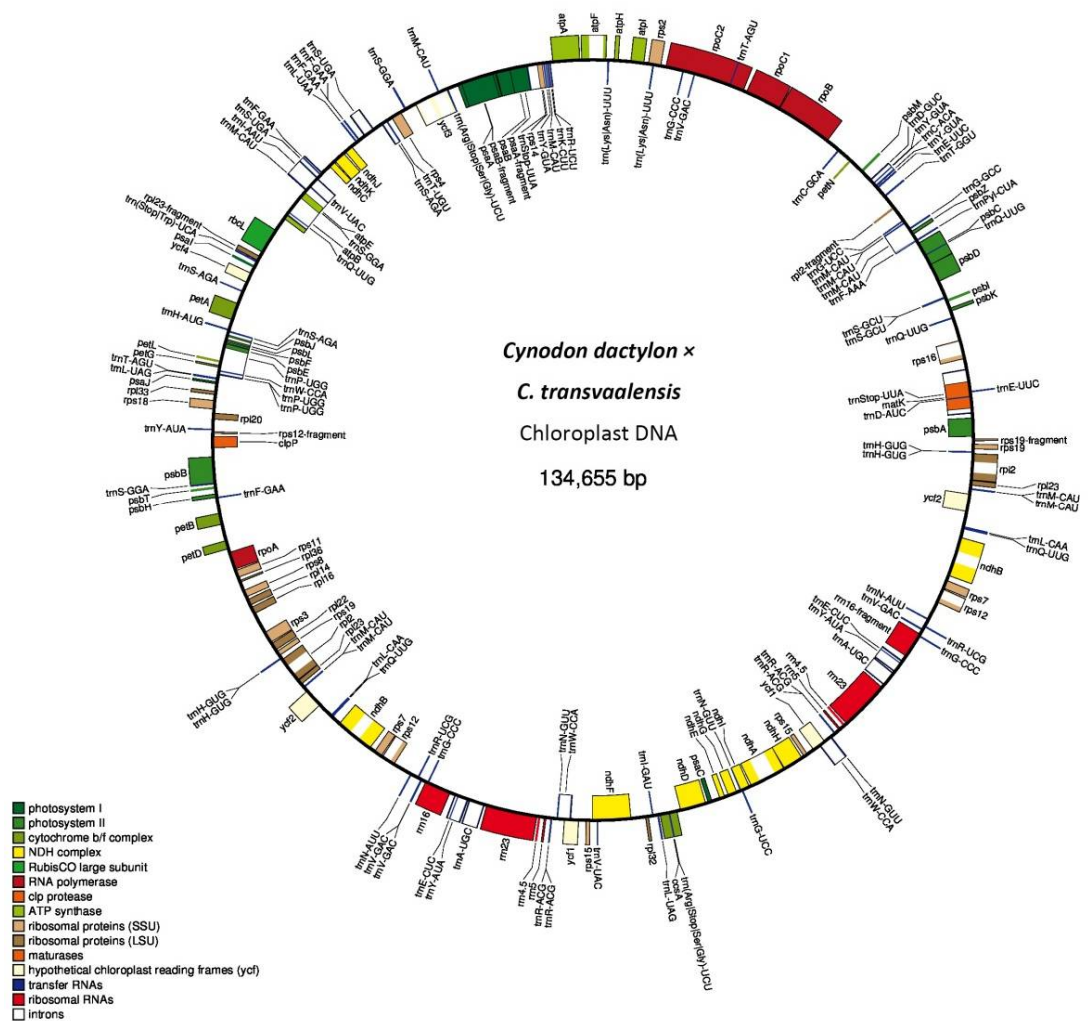

Fig. 1. Gene map of the Cynodon dactylon $\times$ C. transvaalensis chloroplast genome. Genes shown on the inside of the circle are transcribed clockwise, and genes outside are transcribed counter-clockwise. Genes belonging to different functional groups are color-coded.

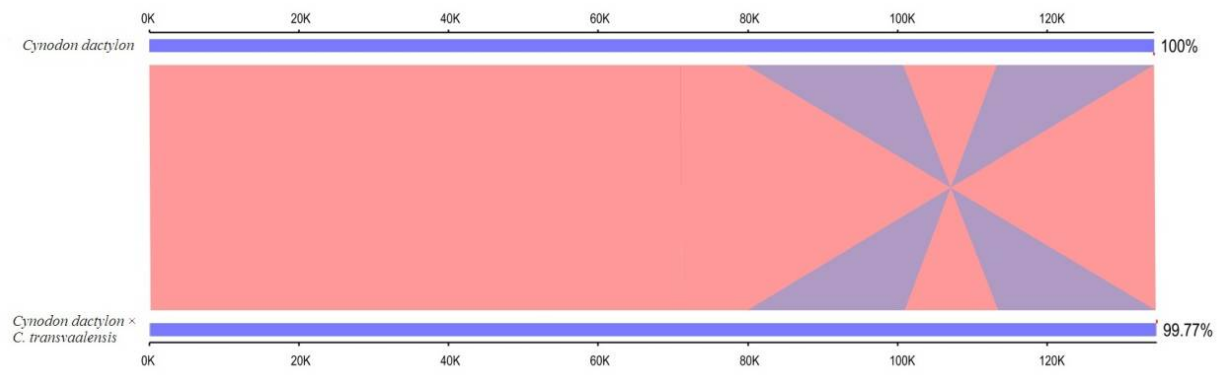

Fig. 2. Comparative analysis between Cynodon dactylon and Cynodon dactylon $\times$ C. transvaalensis. Dark blue region represents collinear sequences, red region indicates positive collinearity, light blue indicates negative collinearity.

Nuclear genes are involved in the regulation of chloroplast gene expression. NEP polymerase, which is encoded by nuclear gene is responsible for the expression of rRNAs, tRNAs, clpP and accD and so on (Krause et al. 2000). Specific nuclear products can affect the processing and stability of specific mRNA transcripts. The mutation of single nuclear gene in Chlamydomonas 
also affects the stability of specific chloroplast mRNA (Gruisserm and Zwrawski 1985). Barkan (1989) thought nuclear encoded proteins may be related to the editing activity of plastid transcripts.

Table 2. List of genes in the Cynodon dactylon $\times$ C. transvaalensis chloroplast genome.

\begin{tabular}{|c|c|c|}
\hline $\begin{array}{l}\text { Gene } \\
\text { category }\end{array}$ & $\begin{array}{l}\text { Groups of } \\
\text { genes }\end{array}$ & $\begin{array}{l}\text { Name of } \\
\text { genes }\end{array}$ \\
\hline \multirow{5}{*}{$\begin{array}{l}\text { Self-repli- } \\
\text { cation }\end{array}$} & DNA-dependent RNA polymerase & rpoA, rpoB, rpoC1, rpoC2 \\
\hline & $\begin{array}{l}\text { Large subunit of ribosomal } \\
\text { proteins }\end{array}$ & $\begin{array}{l}\mathrm{rpl} 2^{\mathrm{a}, \mathrm{b}}, \operatorname{rpl} 14, \mathrm{rpl} 6^{\mathrm{a}}, \mathrm{rpl} 20, \operatorname{rpl} 22, \mathrm{rpl} 23^{\mathrm{b}}, \mathrm{rpl} 32, \\
\mathrm{rpl} 33, \operatorname{rpl} 36\end{array}$ \\
\hline & rRNA genes & $\operatorname{rrn} 16^{\mathrm{b}}, \operatorname{rrn} 23^{\mathrm{b}}, \operatorname{rrn} 4.5^{\mathrm{b}}, \operatorname{rrn} 5^{\mathrm{b}}$ \\
\hline & $\begin{array}{l}\text { Small subunit of ribosomal } \\
\text { proteins }\end{array}$ & $\begin{array}{l}\operatorname{rps} 2, \operatorname{rps} 3, \operatorname{rps} 4, \operatorname{rps} 7^{\mathrm{b}}, \operatorname{rps} 8, \operatorname{rps} 11, \operatorname{rps} 12^{\mathrm{b}}, \operatorname{rps} 14, \\
\operatorname{rps} 15^{\mathrm{b}}, \operatorname{rps} 16^{\mathrm{a}}, \operatorname{rps} 18, \operatorname{rps} 19^{\mathrm{b}}\end{array}$ \\
\hline & tRNA genes & 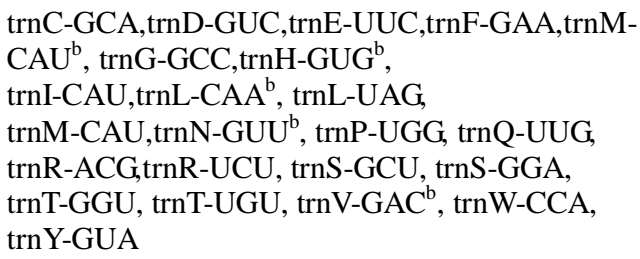 \\
\hline \multirow{6}{*}{$\begin{array}{l}\text { Photo- } \\
\text { synthesis }\end{array}$} & ATP synthase & atpA, atpB, atpE, atpF ${ }^{\mathrm{a}}$, atpH, atpI \\
\hline & Cytochrome b6/f complex & petA, petB ${ }^{\mathrm{a}}$, pet $\mathrm{D}^{\mathrm{a}}$, petG, petL, petN \\
\hline & NADH oxidoreductase & $\begin{array}{l}\text { ndh } \mathrm{A}^{\mathrm{a}}, \mathrm{ndhB}^{\mathrm{a}, \mathrm{b}}, \text { ndhC, ndhD, ndhE, ndhF, ndhG, } \\
\text { ndhH, ndhI, ndhJ, ndhK }\end{array}$ \\
\hline & Photosystem I & psaA, psaB, psaC, psaI, psaJ \\
\hline & Photosystem II & $\begin{array}{l}\text { psbA, psbB, psbC, psbD, psbE, psbF, psbH, psbI, } \\
\text { psbJ, psbK, psbL, psbM, psbN, psbT, psbZ }\end{array}$ \\
\hline & Rubisco & $\mathrm{rbcL}$ \\
\hline \multirow{6}{*}{$\begin{array}{l}\text { Other } \\
\text { genes }\end{array}$} & Conserved open reading frames & ycf4 \\
\hline & c-type cytochrome synthesis gene & $\operatorname{ccs} \mathrm{A}$ \\
\hline & Envelope membrane protein & cemA \\
\hline & Maturase & matK \\
\hline & Protease & $\operatorname{clpP}$ \\
\hline & Translation initiation factor & $\inf A$ \\
\hline
\end{tabular}

${ }^{\mathrm{a}}$ Genes containing introns; ${ }^{\mathrm{b}}$ Duplicated gene (Genes present in the IR regions)

Some proteins encoded by the nuclear gene can interact with the specific stem-and-loop structure of the 5 'end of the mRNA. The composite can change the binding capacity of mRNA and ribosome, which activates or depresses translation (Gillham et al. 1994, Hirose and Sugiura 1996 and Rochaix 1996). Although plastid DNA can encode the protein needed, most of its protein is encoded by nuclear genes. For example, chlorophyll a-b binding protein (cab) and small subunit of RuBisCO are encoded by nuclear gene (Schreier et al. 1985). Besides, changes of DNA structure (Mullet 1988) and selective DNA methylation (Ngernprasirtsir et al. 1989) may also be the mechanisms of chloroplast gene transcription regulation.

\section{Acknowledgments}


This study was supported by the Fund of Guangxi Key Laboratory of Plant Conservation and Restoration Ecology in Karst Terrain (No.17-259-23) and Basic Business Expenses Project of Guangxi Institute of Botany (Guizhiye 17004).

\section{References}

Barkan A 1989. Tissue-dependent plastid RNA splicing in maize: transcripts from four plastid genes are predominantly unspliced in leaf meristems and roots. Plant Cell 1: 437.

Benson G 1999. Tandem repeats finder: A program to analyze DNA sequences. Nucleic Acids Res. 27: 573-580.

BIG Data Center Members 2017. The BIG Data Center: from deposition to integration to translation. Nucleic Acids Res. 45: D18-D24.

Gillham NW, Boynton JE and Hauser CR 1994. Translational regulation of gene expression in chloroplast and mitochondria. Annu. Rev. Genet. 28: 71-73.

Gruisserm W and Zwrawski G 1985. Analysis of promoter regions for the spinach chloroplast $r b c \mathrm{~L}$, atpB and $p s b$ A genes. EMBO J. 4: 3375 .

Harlan JR, de Wet JMJ and Rawal KM 1970. Origin and distribution of the seleucidus race of Cynodon dactylon (L.) Pers. var. dactylon (Gramineae). Euphytica 19: 465-469.

Hanna W 1998. The future of bermudagrass. Golf Course Manage 66: 49-52.

Hirose T and Sugiura M 1996. Cis-acting elements and trans-acting factors for accurate translation of chloroplast psbA mRNAs: development of an in vitro translation system from tobacco chloroplasts. EMBO J. 15: 1687-1695

Hu L, Wang Z and Huang B 2009. Photosynthetic responses of bermudagrass to drought stress associated with stomatal and metabolic limitations. Crop Sci. 49: 1902-1909.

Hu L, Wang Z, Du H and Huang B 2010. Differential accumulation of dehydrins in response to water stress for hybrid and common bermudagrass genotypes differing in drought tolerance. J. Plant Physiol. 167: 103-109.

Huang YY, Cho ST, Haryono M and Kuo CH 2017. Complete chloroplast genome sequence of common bermudagrass (Cynodon dactylon (L.) Pers.) and comparative analysis within the family Poaceae. Plos. One 12: p. eo179055.

Krause K, Maier R M, Kofer W, Krupimska K and Herrmann RG 2000. Disruption of plastid-encoded RNA polymerase genes in tobacco: expression of only a distinct set of genes is not based on selective transcription of the plastid chromosome. Mol. Gen. Genet. 26: 1022.

Kurtz S, Choudhuri JV, Ohlebusch E. Schleiermacher C, Stoye J and Giegerich R 2001. REPuter: The manifold applications of repeat analysis on a genomic scale. Nucleic Acids Res. 29: 4633-4642.

Li R, Li Y, Kristiansen K and Wang J 2008. SOAP: short oligonucleotide alignment program. Bioinformatics 24: 713-714.

Li R, Zhu H, Ruan J, Qian W, Fang X, Shi Z, Li Y, Li S, Shan G, et al. 2010. De novo assembly of human genomes with massively parallel short read sequencing. Genome Res. 20: 265-272.

Lohse M, Drechsel O, Kahlau S and Bock R 2013. Organellar Genome DRAW - A suite of tools for generating physical maps of plastid and mitochondrial genomes and visualizing expression data sets. Nucleic Acids Res. 41: W575-W581.

Porebski S, Bailey LG and Baum BR 1997. Modification of a CTAB DNA extraction protocol for plants containing high polysaccharide and polyphenol components. Plant Mol. Bio. Rep. 15: 8-15.

Mullet JE 1988. Chloroplast development and gene expression. Ann. Rev. Plant Physiol. Plant Mol. Biol. 39: 475-502.

Ngernprasirtsir J, Chollet R and Kobayashi H 1989. DNA methylation and the differential expression of C4 photosynthesis genes in mesophy II and bundle sheath cells of greening maize leaves. J. Biol. Chem. 244: 8241. 
Rochaix JD 1996. Post-transcriptional regulation of chloroplast gene expression in Chlamydomonas reinhardtti. Plant Mol. Biol. 32: 327-341.

Schattner P, Brooks AN and Lowe TM 2005. The tRNAscan-SE, snoscan and snoGPS web servers for the detection of tRNAs and snoRNAs. Nucl Acids Res. 33(suppl_2): W686-W689.

Schreier PH, Seftor EA, Schell J and Bohnert HJ 1985. The use of nuclear-encoded sequences to direct the light-regulated synthesis and transport of a foreign protein into plant chloroplasts. EMBO J4: 25.

Wyman SK, Jansen RK and Boore JL 2004. Automatic annotation of organellar genomes with DOGMA. Bioinformatics 20: 3252-3255.

Zhang T, Zeng CX, Yang JB, Li HT and Li DZ 2016. Fifteen novel universal primer pairs for sequencing whole chloroplast genomes and a primer pair for nuclear ribosomal DNAs. J. Syst. Evol 54: 219-227.

Zhao Y, Du H, Wang Z and Huang B 2011. Identification of proteins associated with water-deficit tolerance in C4 perennial grass species, Cynodon dactylon $\times$ Cynodon transvaalensis and Cynodon dactylon. Physiol. Plantarum 141: 40-55.

(Manuscript received on 29 June, 2018; revised on 15 January, 2019) 